\title{
Direct electrical shock on a leadless pacemaker
}

\author{
Sandrine Venier ${ }^{1} \cdot$ Peggy Jacon $^{1} \cdot$ Pascal Defaye $^{1}$
}

Received: 3 October 2016 / Accepted: 3 February 2017 / Published online: 10 February 2017

(C) Springer Science+Business Media New York 2017

Keywords Leadless pacemaker - Electrical shock $\cdot$ Cardiac surgery

A 61-year-old man with a history of bradyarrhythmia was implanted with a leadless pacemaker (Nanostim ${ }^{\mathrm{TM}}$, Saint Jude Medical) at the apex of the right ventricle (Fig. 1a). Four months later, the patient was scheduled for a cardiac surgery. A ventricular fibrillation occurred during the surgery at the time of cardiac reperfusion, requiring the completion of two direct electrical shocks with internal paddles (Philips defibrillator ${ }^{\circledR}$ ). The defibrillation attempts used energy of $20 \mathrm{~J}$ applied directly to the fibrillating heart to restore rhythmic contraction. Figure $1 \mathrm{~b}$ illustrates the
Nanostim ${ }^{\mathrm{TM}}$ position after surgery. The device was interrogated just after the surgery: No error message occurred and pacing, sensing, and communicating functions were as effective as previous controls. The pacemaker was in VVI mode with a basic rate of $50 \mathrm{bpm}$, a pulse amplitude and duration of $2.5 \mathrm{~V}$ and $0.4 \mathrm{~ms}$, and a R-wave sensitivity of $2 \mathrm{mV}$. The first EKG recorded in the recovery room after the surgery is presented in Fig. 1c. The patient was in AF with intermittent paced QRS. The electrical cardioversion did not cause the pacemaker to reset. For transvenous pacemaker, the AHA recommends positioning the external defibrillation pad at least $1 \mathrm{in} .(2.5 \mathrm{~cm})$ away from the device [1]. To the best of our knowledge, this is the first case of an internal cardioversion on a leadless pacemaker during a cardiac surgery.
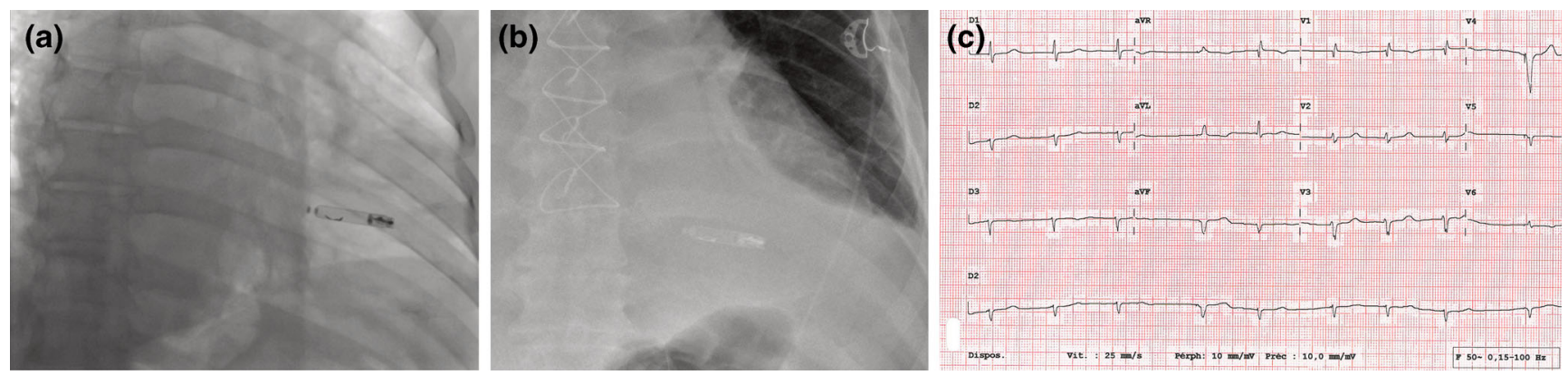

Fig. 1 a The leadless pacemaker at the apex of the right ventricle one day after the implantation. $\mathbf{b}$ The Nanostim ${ }^{\mathrm{TM}}$ position after the cardiac surgery. $\mathbf{c}$ The first EKG recorded in the recovery room after the surgery with intermittent paced QRS

Sandrine Venier

svenier@chu-grenoble.fr

1 Electrophysiology Service, University Hospital of Grenoble Alpes, CS 10217, 38043 Grenoble Cedex 9, France

\section{Reference}

1. Ecc Committee S, Task Forces of the American Heart A. 2005 American Heart Association guidelines for cardiopulmonary resuscitation and emergency cardiovascular care. Circulation. 2005;112: IV1-203. 